\title{
Comment on "Analysis of Pharmacokinetic and Pharmacodynamic Parameters in EU-Versus US-Licensed Reference Biological Products: Are In Vivo Bridging Studies Justified for Biosimilar Development?"
}

\author{
Christopher J. Webster ${ }^{1}$ - Gillian R. Woollett ${ }^{2}$ (D)
}

Published online: 1 August 2019

(c) The Author(s) 2019

\section{Dear Editor,}

We wish to respond to commentary about our paper [1], "A 'Global Reference' Comparator for Biosimilar Development," made in the article by Tu et al. [2] recently published in BioDrugs.

In our paper, we set out a case for bridging studies between versions of a biosimilar reference product approved in different jurisdictions (most notably, the EU and the USA) to be not required when a sponsor can substantiate from information already in the public domain that both versions were approved based on at least some of the same phase III clinical studies. We proposed an evidentiary basis for the acceptance of a foreign version of a reference product as a comparator in permitted studies to establish biosimilarity that is much simpler and less costly than that expected currently in these jurisdictions. Further, we showed that this principle can be generalized to permit any version of the reference product approved in a "highly regulated" jurisdiction to serve as a comparator in permitted studies in all other jurisdictions-hence, the concept of the "global comparator".

We commend Tu et al. [2] for their paper, which confronts the same problem and supports by careful data analysis our contention that costly and duplicative studies are unnecessary to qualify a comparator for permitted studies when the terms of our proposed evidentiary basis are satisfied. However, in their comments on our paper, the authors stated,

"Post-approval manufacturing changes or production site transfers were tightly controlled by regula-

Gillian R. Woollett

GWoollett@Avalere.com

BioApprovals, Acton, MA, USA

2 FDA Strategy and Regulatory Policy, Avalere Health Inc., Washington, DC, USA tory authorities and the consistency between pre- and post-changed products were evaluated using the same principles and guidelines (e.g., International Conference on Harmonisation Q5E) adopted by most countries worldwide. According to the arguments of Webster and Woollett, there was no reason to believe there would be any distinct variations among different sources of reference biological products.

However, although rare, there is a small possibility that differences may occur among reference biological product sources".

This is a mis-statement of our case, which is not based on a tenuous "no reason to believe" that there could be variations in reference products from different sources. While we believe that the comparability data submitted to justify manufacturing changes are a potent and effective assurance of product consistency, as evidenced by the thousands of manufacturing changes that have been accepted on this basis, we accepted fully that differences in biologics are possible but argued conclusively that "any quality differences between the two product versions can be considered inconsequential as regards their use as comparators for biosimilar development".

The reason for our confidence is that, in both the USA and the EU, the only studies of a biosimilar for which a foreign version of the reference may be permitted as a comparator are certain nonclinical and clinical studies (particularly, powered efficacy and pharmacodynamic studies and in vivo nonclinical studies) that are intended to resolve any "residual uncertainty" as to biosimilarity, rather than to evaluate precisely the critical analytical and functional differences between a biosimilar and its reference. All these latter "pivotal" studies-including comprehensive analytical characterizations, in vitro functional studies and human pharmacokinetic (PK) studies-are required to be conducted against the locally sourced version of the reference [3, 4], 
and foreign versions of the reference are currently not eligible as comparators.

The accumulated experience with biosimilars approved to date shows that, for reference products approved in both jurisdictions based on the same clinical data, not only has bridging between the US and EU versions of the reference never failed but also biosimilars that have been shown to be analytically highly similar to the local version of the reference have never failed to meet their primary equivalence endpoints when compared in powered efficacy studies or pharmacodynamic studies against the foreign version of the reference. Clearly, the reason for this has nothing to do with the fact that bridging studies have been conducted but is explained by two other circumstances:

(i) The separate versions have a common antecedent and are known to be sufficiently alike to have been approved in each of the jurisdictions on the same clinical data.

(ii) The studies in which a foreign comparator is permitted are well-known to be relatively insensitive to minor differences in the composition of biologics, so any such differences would not be reflected in the outcomes of these studies.

The authors' own paper adds valuable support to this conclusion. Particularly significant is the finding that, in two of the three cases in which the PK variables of the two versions did not fall within the acceptance range at the first attempt, they did so in a second study, and that in the third case, the marginal nonconformity was due to inherent hypervariability that was also observed by other investigators with a different method of administration.

To summarize, in our view, the reason why elaborate studies are unnecessary, and actually superfluous, when it is known that the two versions of the reference have been approved on the same clinical data is not because quality differences may not exist but because they will make no difference in the studies for which a foreign comparator is permitted in the EU and USA. Since it is clearly necessary to document an objective relationship between the reference product versions in order to qualify the foreign version as a proxy for the local version, the substantiated fact that both versions were approved on the same clinical data serves that purpose.

If our view is accepted, a further question may arise. What is gained by a removal of the requirement for bridging data if comprehensive analytical and human PK data comparing a biosimilar candidate to the local version of the reference must be provided in the biosimilar marketing application, as is the case in the EU and USA? In practice, most sponsors will choose to study their candidate biosimilars against the EU and US reference versions in parallel, so they will typically have access to the data required for bridging between these jurisdictions. In our view, the advantage of the "global comparator" concept is that it allows much more practical flexibility in the conduct of any studies that may be required in order to resolve "residual uncertainty" as to the biosimilarity of a biosimilar candidate. These could be conducted at times and places that are not available under the requirement for bridging data. Also, the requirement for bridging data is of a piece with, and supports, the requirements in some jurisdictions for a local clinical trial of biosimilars. In our view, local clinical studies of biosimilars are not scientifically justified if the reference product has been licensed in the jurisdiction.

We welcome further comments but, absent any, would like to see such an approach as we have proposed become the norm for regulators to facilitate further biosimilar entrants and greater competition in all markets. This will create the headroom for the next generation of originator biologics and drugs.

\section{Compliance with Ethical Standards}

Funding No sources of funding were used to conduct this study or prepare this manuscript.

Conflict of interest CJW has no conflicts of interest that are directly relevant to the content of this manuscript. GRW is employed by Avalere Health, an advisory services company that works across the breadth of healthcare but neither advocates nor lobbies for any client.

Open Access This article is distributed under the terms of the Creative Commons Attribution-NonCommercial 4.0 International License (http://creativecommons.org/licenses/by-nc/4.0/), which permits any noncommercial use, distribution, and reproduction in any medium, provided you give appropriate credit to the original author(s) and the source, provide a link to the Creative Commons license, and indicate if changes were made.

\section{References}

1. Webster CJ, Woollett GR. A 'global reference' comparator for biosimilar development. BioDrugs. 2017;31(4):279-86. https:// doi.org/10.1007/s40259-017-0227-4.

2. Tu C-L, Wang Y-L, Hu T-M, Hsu L-F. Analysis of pharmacokinetic and pharmacodynamic parameters in EU versus US-licensed reference biological products: are in vivo bridging studies justified for biosimilar development? BioDrugs. 2019;33(4):437-46. https ://doi.org/10.1007/s40259-019-00357-2.

3. Guidance for industry: quality considerations in demonstrating biosimilarity of a therapeutic protein product to a reference product. US Food and Drug Administration. 2015. p. 9.

4. Committee for Medicinal Products for Human Use (CHMP): guideline on similar biological medicinal products CHMP/437/04 Rev 1, October 2014, Section 3.2. 